\title{
PATTERN APPLICATION VS. INHERITANCE IN SDL
}

\author{
Birgit Geppert, Frank Rößler, Reinhard Gotzhein \\ University of Kaiserslautern, P.O. Box 3049, 67653 Kaiserslautern, Germany \\ \{geppert, roessler, gotzhein\}@informatik.uni-kl.de
}

\begin{abstract}
Recently, SDL patterns have been introduced as a new concept to increase reusability in system design that is based on the formal description technique SDL. An integral part of this approach is the notion of pattern application, comprising the selection, adaptation, and composition of SDL patterns with a (possibly empty) specification context. We argue that the application of SDL patterns is a general form of specialization, supporting the reusability of design decisions, and compare it to the well-known reuse mechanism of inheritance in SDL.
\end{abstract}

\section{INTRODUCTION}

Inheritance is one of the key mechanisms of object-oriented system development to support reusability of design and implementations. It is based on the concept of specialization, a (formal) relation between classes. Though being a semantic concept, specialization is mostly captured syntactically in existing language approaches including formal description techniques (FDTs). This has the advantage that it is straightforward to use it as a basis for defining inheritance, and thus to apply this concept in practice.

Previous work $[4,5]$ distinguishes between different kinds of inheritance. Incremental inheritance is the process of adding or redefining attributes to/of an existing class definition, yielding new class definitions. However, there is no guarantee that the objects of the newly-derived class can replace objects of the parent class. Subtyping inheritance, on the other hand, ensures that objects of a subclass are also members of the corresponding superclass. Thus, the substitutability constraint is met. Subtyping inheritance of dynamic objects has been studied, for instance, in the context of CSP or Object Z.

Though having a semantic foundation, subtyping inheritance does not by itself support reusability during software development. On the other hand, incremental inheritance, while boosting reusability, does not meet the substitutability constraint.

\footnotetext{
* This work is supported by the Deutsche Forschungsgemeinschaft (DFG) as part of the Sonderforschungsbereich SFB 501 Development of Large Systems with Generic Methods.
} 
Therefore, some attempts have been made to find restricted forms of incremental inheritance that meet this constraint, thus implying subtyping inheritance. Some results in this direction are reported in [4] (CSP), [5] (Object Z) and [13] (Estelle). From these results, it can be concluded that though being very elegant from a systematical point of view, the demand for substitutability strongly limits the applicability of incremental inheritance and thus reusability. Therefore, object-oriented languages usually support incremental inheritance without taking subtyping inheritance into account.

In this paper, we consider incremental inheritance in the context of SDL (Specification and Description Language) $[7,14,16]$. SDL is a formal description technique for distributed systems in general and communication systems in particular. It has been developed and standardized by ITU-T, the International Telecommunications Union, and is widely used in industry and academia. SDL has several graphical language elements, which improve readability of both system structure and component behavior. A further part of the industrial success of SDL is attributed to the existing commercial tool environments, supporting all phases of system development with SDL including analysis, simulation, and code generation.

In $[9,11]$ we present the SDL pattern approach, which integrates the design pattern concept $[2,8]$ with SDL. Generally speaking, SDL patterns describe generic solutions for recurring design problems, which can be customized for a particular.context. While conventional design patterns are specified independent of a possible design language, it is assumed that the target language for SDL pattern instantiation is SDL. Thereby, we benefit from the formal basis provided by SDL, so that SDL patterns are actually characterized as formalized design patterns. Instead of specifying and applying the patterns rather informally, a formal target language such as SDL offers the possibility of precisely specifying how to apply a specific pattern, under which assumptions this will be allowed, and what properties result for the embedding context. This is a major improvement as compared to conventional design patterns, which mainly rely on natural language-based pattern description and, to a large degree, must still leave pattern application to the personal skills of the system designer. An integral part of the SDLpattern approach is the notion of pattern application, comprising the selection, adaptation, and composition of SDL patterns with a (possibly empty) specification context. We argue that the application of SDL patterns is a general form of specialization, supporting the reusability of design decisions, and compare it to the well-known reuse mechanism of inheritance in SDL.

The paper is organized as follows. First, we survey some object-oriented language constructs of SDL, with focus on inheritance, and summarize the SDL-pattern approach. Then, we elaborate on the notion of pattern application, which is an integral part of the SDL-pattern approach, and argue why we consider pattern application a particular form of specialization. We then compare pattern application and inheritance and finally draw conclusions.

\section{OBJECT-ORIENTATION IN SDL}

An SDL specification [7, 14, 16] is a formal description of both the architecture and the behavior of a system. Systems are hierarchically structured into blocks connected 
by channels, which can in turn be decomposed into blocks or processes. While blocks are used for structuring purposes only, processes are assigned dynamic behavior consisting of internal actions and interaction by asynchronous signal exchange with other processes or the system environment. An SDL system is conceptually modeled as a set of asynchronously communicating extended finite state machines (EFSMs).

The starting point for the SDL language development dates back to 1972. In several steps, the language has been extended, and in 1988, a formal semantics was defined. In 1992, a major revision of SDL led to the addition of object-oriented language constructs, making SDL an object-oriented design language. We will now survey these constructs as far as it is needed for the comparison of SDL-pattern application and SDL inheritance.

Basic object-oriented concepts are object and class. For historical reasons, these concepts are named differently in SDL, the corresponding notions being instance and type. Examples of SDL instances are system, block, process, service, procedure, and signal. SDL instances have an identity, e.g., an explicit process id, and are characterized by attributes such as structure, behavior, or values. Sets of instances can be defined by SDL types, e.g., block type, process type, service type, data type etc. SDL types can be specialized, leading to subtypes. The specialization of a type A can lead to additional attributes, i.e., structure, behavior, or data, as well as to the redefinition of existing attributes. SDL distinguishes between simple specialization (adding attributes) and advanced specialization (redefining attributes). Syntactically, specialization is supported by an inheritance mechanism. In the following, we consider the specialization of behavior only; for further details, especially on specializing structure, see $[7,16]$.

Figure 1 gives examples of specialization by adding and/or replacing behavior. By inheriting from P1Type, P2Type acquires all attributes (declarations, major states, transitions, gates etc.) from P1Type. Further attributes extending the process behavior, in particular a new transition (and possibly additional major states) and an extension of gate $\mathrm{g} 1$, are added. Note that in the definition of P1Type, the input transition accepting sig1 is declared as virtual. This allows the redefinition of this transition in process types that inherit P1Type as shown in P3Type (see Figure 1). If a virtual transition is redefined, it is replaced by another transition. A redefined transition is again virtual by default unless it is marked as finalized. In general, certain constraints apply to the redefinition of virtual transitions. For instance, if the virtual transition is an input tran-
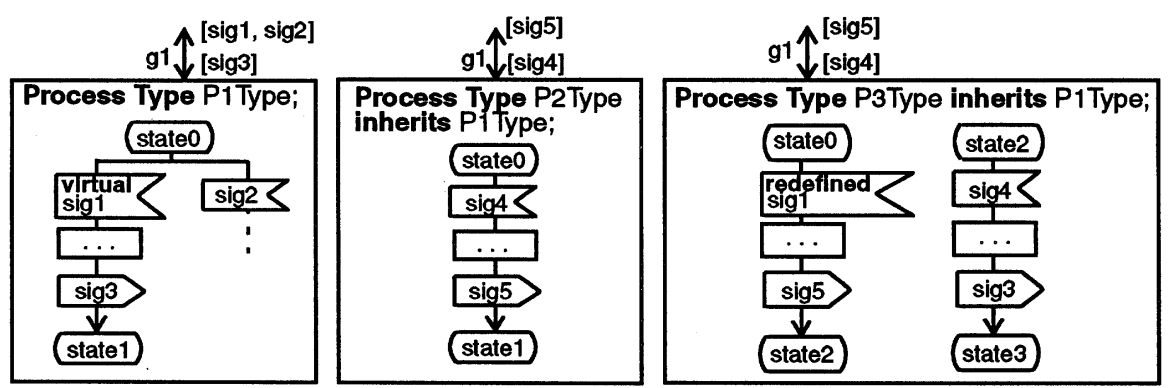

Figure 1. Specialization of behavior in SDL 
sition, the redefined transition must be an input transition, a priority input transition, or a save construct; virtual spontaneous transitions must be redefined into spontaneous transitions, etc.

Further specialization is possible in SDL with respect to structure. For instance, a block type A specializing a block type B inherits all attributes, i.e., all declarations, instantiations, connections, of B and can add further attributes. As in case of transitions, block types or process types may be declared as virtual, which allows one to replace this block type in a specialization of the containing class specification. This option is necessary in cases where the interaction of added instances with inherited instances influences their behavior. Also, signals can inherit attributes of other signals while adding further signal parameters. Finally, we note that SDL does not support multiple inheritance.

\section{THE SDL-PATTERN APPROACH}

This section summarizes concepts for a construction set of protocol building blocks from which a protocol designer can select SDL patterns and configure them to a customized, formal protocol specification. In order to get highly flexible building blocks and to increase the quality of resulting products, we combine the design pattern approach with SDL. For further details, the reader is referred to $[9,11]$.

\section{SDL patterns and methodology}

Scattered parts of a given SDL specification together may perform a certain functionality. By analysis, abstraction, and documentation, such a design solution can be reused whenever the design problem arises again. This is the main idea of software patterns in general and SDL patterns in particular. Roughly speaking, an SDL pattern is characterized as a reusable software artifact that represents a generic solution for a recurring design problem. It is assumed that SDL be the applied design language. SDL patterns serve three main purposes:

- First, SDL patterns are descriptive in nature. That is, they describe design expertise and experiences gained in prior projects and allow to pass this knowledge on to other developers. A collection of patterns can therefore be seen as some kind of textbook.

- SDL patterns generate a design solution for a given problem. Therefore, the solution is described by an SDL fragment that consists of several syntactical elements (e.g., transition fragments), which may be scattered over the context specification. Additionally, application guidelines describe when the pattern could be applied and how to adapt the SDL fragment and compose it with the given context specification.

- Furthermore, SDL patterns help to document a given design. The information about embedded pattern instances can be inserted during pattern-based design, i.e., when applying a pattern (top-down documentation). However, pattern information can also be inserted into a given SDL specification by pattern-based reverse engineering (bottom-up documentation). Therefore the SDL specification is scanned for pattern instances and enriched with suitable pattern comments. 
Name. The name of the paltern, which hould intuitively describe its purpose.

Intent a short infonnal description of the particular design problen and its solution.

Motivation, Ar example ( trom the area of conniningation systens) where the design prob. lem arises. This is appropriale for lilustrating the relevance and need of the pattern.

Strueture. A graphical representation of he structiral aspects of the design solution ising an GMI objed model. This defrines the involved design elements and thelr relations.

Message scenario. Examples scenarios lilustrating typical interactions bet ween the invol ved objects (e g. protocolentities, service users, service providers, protocol functions) are spec. ified by using $165 C$ diagroms.

S11 f agment. The mere syntactical part of the design solution is defined by a generic SDI frog ment which is adapted and syotactically enbedded w ben apply ing be pattern $1 \mathrm{f}$ nore than one SDI version of the design solution s, possible (realization as SDI service or procedure, interaction by message passing or shared variables, etc.), fragments for the nost fre quent versions are inclided, 6 or ead fragnent, corres ponding syntactical embedding hiles are defined in terins of the SDI s yotax.

- Rules for retaming of the generic identifiers of the SDI fragment

- Rules for composing the pattern instance with the enbed ding context This could, for Instance, result in the addition of new transitions or SDI ser Vices or in a refinement of existing ypes or transitions.

Note that an SDI fragment s generally uol a s ytactically complete SDI specification but a fragment, which has to be adapted and composed with an embedding context f Fron o seman tic point of yiew. SDI fragments therefore only becone formally theaning ful when they are part of a complete specification.

Semantic properties, e teperties of the resulting specification that are introduced by the ent. bedded pattern. This also neludes a description of assunptions under which these properlies. hold Care has to betaken not to destroy a patrerns assumptions during firther development steps, e.8. by manipulating the context specification. The semantic properties define the pat tern's intent nore precisely.

Refinement, Ar embedded pattern instance can be fullier refined, e, g, by the embedding of another pattern Instance in subsequent development steps. Refinements compatible with the parten's intent are specified.

Cooperative usage fossible usage with o ther patterns of the pool is described This is feasi ble and especilliy useful for a specific application donain as in our case and distinguishes a pool of SDI patterns from a mere pattern catalogue where the patterns are unrelated of only loosely telated.

\section{Figure 2. SDL-pattern description template}

The specification of an SDL pattern is organized by a standard description template (Figure 2). Its main items are sketched in the following: the syntactical part of the design solution is defined by the generic SDL fragment, which has to be adapted and textually embedded into the context specification when applying the pattern. Adaptation and composition of SDL fragments is prescribed in terms of syntactical embedding rules, which, e.g., guide the renaming of generic identifiers or specialization of embedding design elements. Usually pattern semantics are not completely captured by an SDL fragment. Due to language constraints, this would otherwise result in an overspecification of the design solution and reduce the reuse potential. Thus, additional semantic properties specify preconditions for pattern application as well as behavioral 
changes of the embedding context. Though semantic properties are currently stated in natural language, it is possible to express them precisely in temporal logic. Also, restrictions on the refinement of pattern instances are specified in order to prevent a pattern's intent from being destroyed by subsequent development steps. Figures 6 and 8 show instances of the description template.

In general, the SDL pattern concept is not restricted to any application domain. This means that SDL patterns can be defined for any domain where SDL is applicable and can deal with system architecture or behavior. However, in the SFB 501 project we are concerned with the generic engineering of communication software. Therefore, the current pool of SDL patterns contains building blocks from the domain of communication protocols that deal, for instance, with the interaction behavior of distributed objects (an example is illustrated in Figure 6), error control (lost or duplicated messages, see Figure 8), lower layer interfacing, or dynamic creation of protocol entities. Not included so far are SDL patterns dealing with system architecture. This is a matter of future work.

Along with the standard template for SDL pattern description, we have also defined a process model for the application of SDL patterns. The configuration process suggests an incremental design, where separate protocol functionalities are specified one after the other. During the design activity of each development step, one or more SDL patterns can be applied: based on the current SDL context specification and the protocol functionality to be incorporated, a proper set of SDL patterns is to be selected $^{*}$. As SDL patterns represent generic design solutions, a selected pattern, respectively its corresponding SDL fragment, has to be adapted to seamlessly fit the embedding (context) specification (e.g., generic signal names have to be set according to the pattern's syntactical embedding rules). Finally, the pattern instance is ready to be composed with the embedding specification. Application of patterns in the sense of selection, adaptation, and composition is specifically supported by certain items of the SDL-pattern description template. The result of this design activity is an intermediate SDL design specification, which serves as the context specification for the next development step.

\section{Test projects and tool support}

In order to show the feasibility of the SDL-pattern approach, we conducted several test projects. For instance, we have performed an SDL-pattern based re-engineering of most parts of the Internet Stream Protocol ST2+ [6], resulting in a systematic and formal SDL design specification. Thereby a very large portion of the final ST2+ specification resulted from SDL patterns. In particular, we demonstrated how SDL patternbased design can boost the developer's confidence in the correctness of the resulting product. Another test project was part of a more comprehensive project where a realtime communication subsystem was developed on top of a Controller Area Network (CAN) installation. In [10] we demonstrate how the protocols supporting user communication and certain management tasks were configured using SDL patterns. We also

\footnotetext{
* Note that for some design problems the pool of pre-defined building blocks may not contain an adequate solution. This gives rise to the development of a new SDL pattern or an ad hoc solution.
} 
applied the SDT Cadvanced code generator to implement the resulting design specification on a PC cluster. Again, it turned out that SDL-pattern based configuring of communication protocols yields more systematic designs, i.e., readability and maintainability is improved and less design errors occur, since the design decisions are well founded and documented.

The SDL-pattern approach offers several possibilities for tool support, ranging from search engines for pattern repositories to verification tools for pattern application [3]. Currently, we are developing a prototype SDL-pattern editor, called SPEEDI. SPEEDI supports documentation as well as adaptation and composition of SDL patterns. Implicit documentation of an SDL specification by highlighting embedded pattern instances allows to grasp the elements of a design solution all at once. Readability is further improved by providing a convenient way to navigate through the design and to collapse inspected pattern instances. During adaptation and composition of a pattern, SPEEDI assists in the renaming of generic identifiers and the observation of the pattern's embedding rules.

\section{EXAMPLES OF PATTERN APPLICATION}

So far, we have applied the term pattern application in a rather broad sense, where pattern selection, adaptation, composition, and validation was subsumed. In the following, we restrict the notion of pattern application to the more syntactical subtasks of pattern adaptation and composition. In this sense we give two examples, which shall illustrate how SDL patterns are applied. The examples are taken from a former test project [12], where a real-time communication subsystem was customized in order to control an „inverted pendulum“ simulation over a Token-Ring LAN. The specifications below resulted from the first and third development step, respectively. However, only two of the pattern applications, which finally implemented the procedures for connection establishment, are demonstrated. For reasons of simplicity, other protocol functionalities (e.g., the reservation of sufficient network resources to guarantee a specified quality of service during data transfer) are omitted.

According to our process model, starting point for a pattern application is an SDL context specification and a corresponding design problem. For our first example, the context specification is shown in Figures 3 and 5. Figure 3 illustrates the system archi-

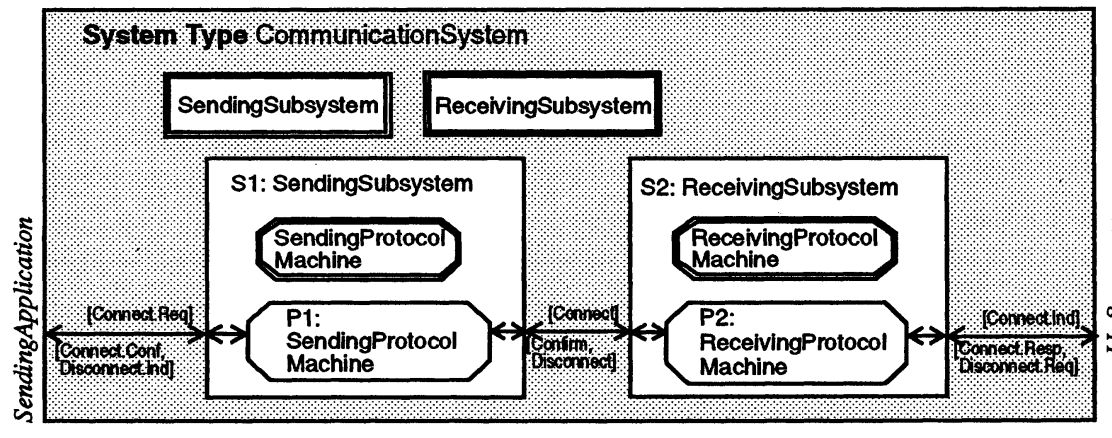

Figure 3. Architectural overview 

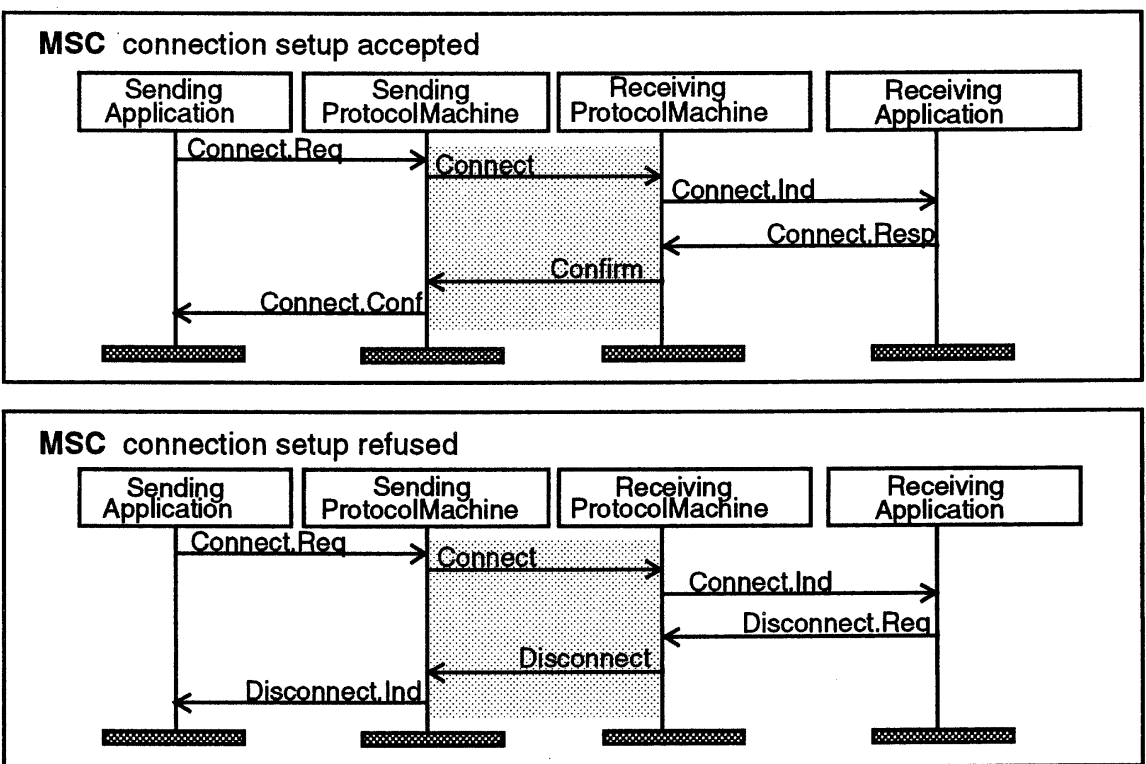

Figure 4. Interaction scenarios for connection setup

tecture, which consists of two communication subsystems - one for the sending side and one for the receiving side. The behavior of each subsystem is specified by its own SDL process. Figure 5 shows the definition of the process types. As can be seen, only communication between SendingApplication and SendingProtocolMachine has been considered so far. Note that SendingApplication and ReceivingApplication are part of the environment and therefore not explicitly modeled.

The design problem to be solved in the current development step is illustrated by the Message Sequence Charts (MSC) [15] of Figure 4: we need to realize three cascaded two-way handshakes between SendingApplication and SendingProtocolMachine, SendingProtocolMachine and ReceivingProtocolMachine, and finally between ReceivingProtocolMachine and ReceivingApplication.
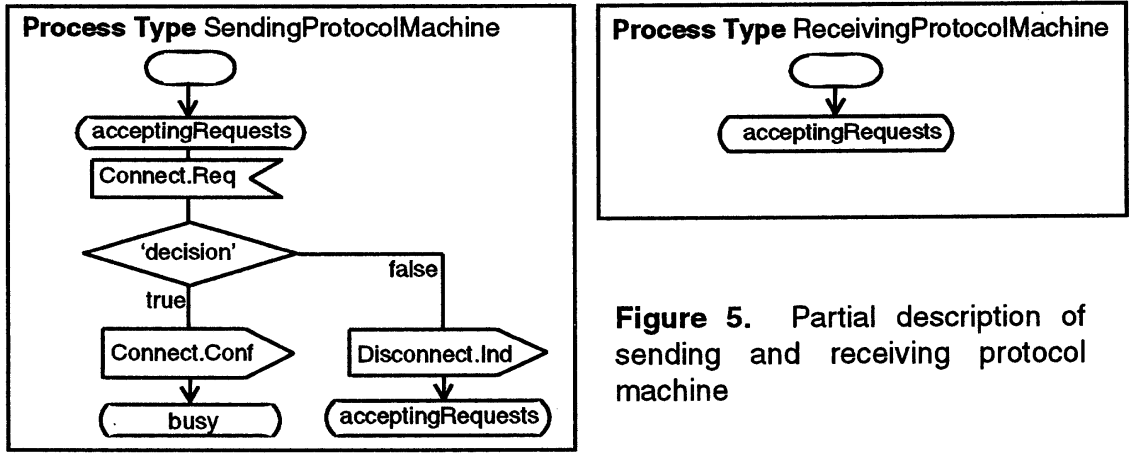

Figure 5. Partial description of sending and receiving protocol machine 


\section{BLOCKINGREQUESTREPUY}

\section{Intent:}

The BlockingRequestReply pattern introduces a confirmed interaction (two way handshake) between two given automata Altomaton $A$ and $A$ utomaton $B$. After a trigget from the embed. ding context. RequestAutomaton $A$ sends a request and is blocked until receiving a reply. The request is eventually received by ReplyAtutomilon B, which replies and finally releases Requestiutonaton $\mathrm{A}$ from its waiting state.

Structure.

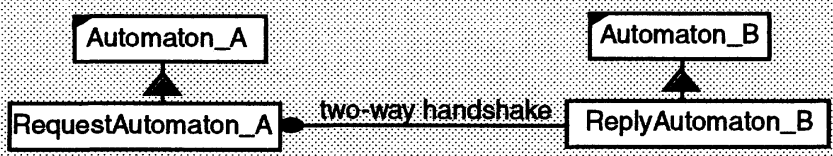

SDV fragment:
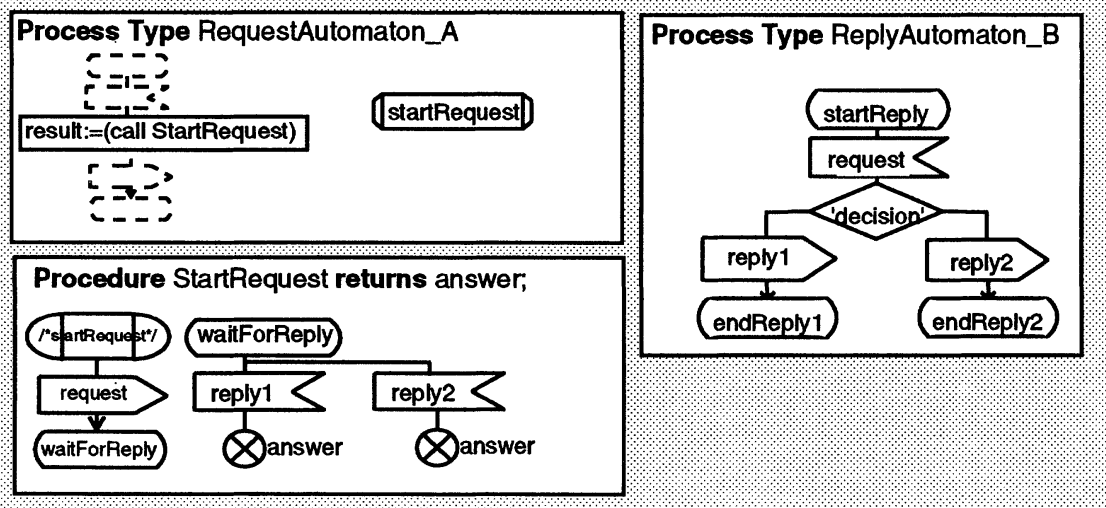

Figure 6. Excerpt of the SDL pattern BlockingRequestReply

To fully implement the described interaction scenario between the four agents, one pattern is applied several times. The pattern that performs the two-way handshake between SendingApplication and SendingProtocolMachine is already embedded into the context specification (Figure 5). The pattern application discussed in the following covers the part shaded grey in Figure 4. We skip the pattern selection activity here and state that this kind of interaction behavior corresponds to the SDL pattern BlockingRequestReply. An excerpt of the pattern is given in Figure 6.

In general, pattern application is a relation between two SDL specifications, which is expressed by an UML [1] object model in combination with an SDL fragment (e.g., see Figure 6) as follows. The design elements of the context specification are marked by the symbol $\boldsymbol{V}$ in the UML object model. The specialization of design elements through pattern application is represented by the relation $\triangle$ called design element specialization (or $d$-specialization for short). During pattern application, all d-specializations are applied simultaneously, leading to the replacement of the original design elements. In addition, new associations may be established through pattern application, which can be represented as usual in the UML model. 

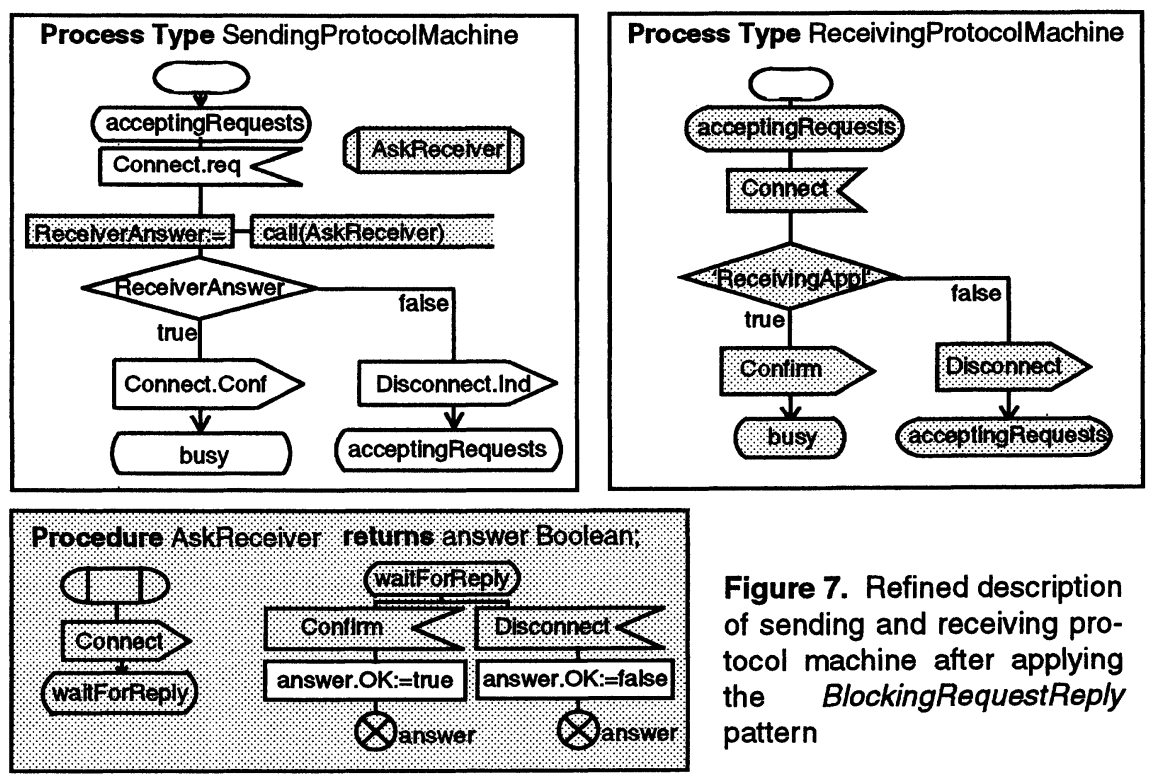

Figure 7. Refined description of sending and receiving protocol machine after applying the BlockingRequestReply pattern

In case of the BlockingRequestReply pattern (Figure 6), the structural diagram shows two design elements (Automaton_A and Automaton_B) participating in the pattern application. The resulting design elements (now called RequestAutomaton_A and ReplyAutomaton_B) are derived as defined by the SDL fragments, and are in a twoway handshake association. In our example, SendingProtocolMachine corresponds to (Request)Automaton_A and ReceivingProtocolMachine plays the role of (Reply)Automaton_B.

Adapting the BlockingRequestReply pattern divides into two activities: firstly, several generic identifiers (e.g., state or signal names) must be renamed in order to fit the context specification. Secondly, some refinements are necessary that complete the behavior of the embedded pattern instance. Note that these refinements characterize variant parts of the design solution generated by the SDL pattern, while invariant parts are captured by the pattern description. The trade-off between variant and invariant parts of the design solution strongly influences the reusability of the pattern. Figure 7 shows the SDL specification after applying the BlockingRequestReply pattern, where the embedded pattern instance is shaded grey. A comparison with the SDL fragment of Figure 6 reveals the applied renaming and refinement steps. For instance, the generic signal names request, reply1, and reply2 have become Connect, Confirm, and Disconnect, respectively, in the embedded pattern instance. Similarly, the informal 'decision' has been refined according to the receiver answer to the connect message. Note that renaming and refinement steps are constrained by the syntactical embedding and refinement rules of the pattern description (not shown in Figure 6). Composition of the pattern instance with the embedding context is also prescribed by syntactical embedding rules. In case of BlockingRequestReply the protocol designer must determine the "triggering point" of the two-way handshake, i.e., the (preexisting) transition of the 


\section{TMERCONTROLUEDREPEAT}

\section{Intent:}

Timer ControlledRepeat extends a confinned message exchang between two design elenents Sendi utomaton and Acknowled geAutomaton for the case of possible nessage losses during data transfer. If an expected acknowledgment does not arive before the expiry of a tiner the iressage is repeated (Positive Acknowledgment with Retransinission). This pattern does rot deal with the problem of message disruption or duplication.

\section{Motivation:}

For a Blocking RequestReply pattern instance, the requester will deadlock, if the reliable trans thission of the request or teply siguals is not guaranteed. Therefore teplies are observed by Timer o nirolledRepear in case of an unreliable basic service.

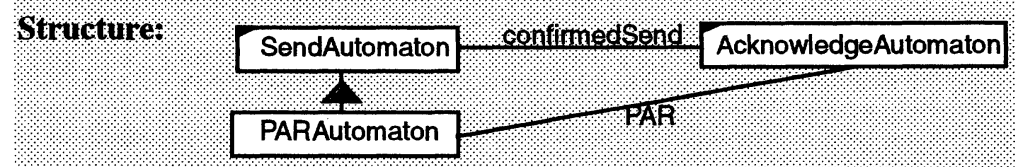

SDI fragment:

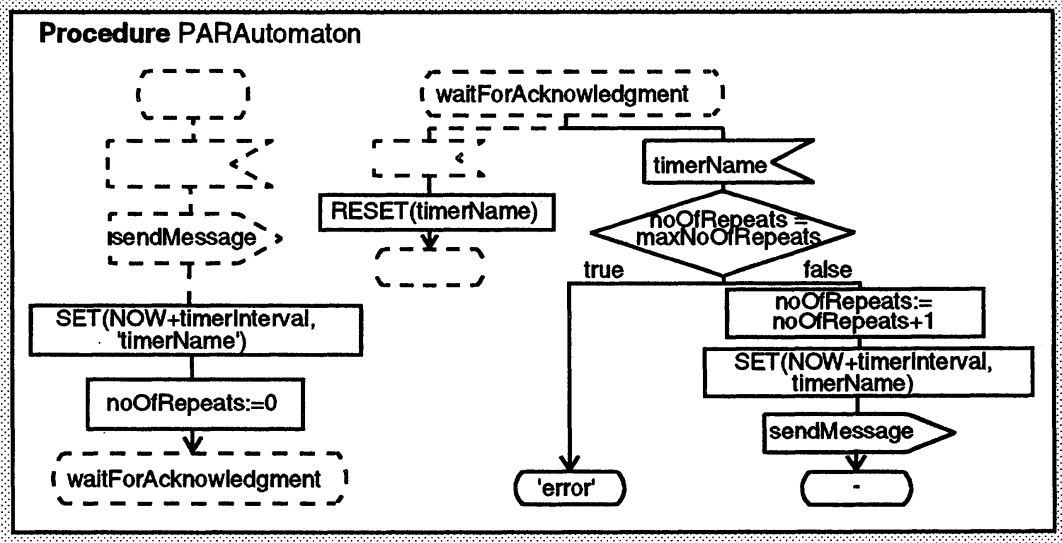

Figure 8. Excerpt of the SDL pattern TimerControlledRepeat

context, where the procedure call should be placed. Apart from that, composition means a textual insertion of the adapted SDL fragment.

We now turn to the second example of an SDL pattern application. Though we skip a complete development step from our former test project, the relevant part of the context specification is already covered by Figure 7 . The design problem we want to solve now is to recover from message losses, i.e., we expect that Connect, Confirm, or Disconnect signals are sometimes lost during data transfer. To cope with lost messages, the TimerControlledRepeat pattern (Figure 8) is applied. TimerControlledRepeat relies on a confirmed message exchange between SendAutomaton and AcknowledgeAutomaton. If an expected reply does not arrive before the expiry of a timer, the message is repeated (Positive Acknowledgment with Retransmission) a maximum number of times. In our context specification AskReceiver corresponds to SendAutom- 
aton/PARAutomaton, while ReceivingProtocolMachine corresponds to AcknowledgeAutomaton. The confirmed message exchange that has to be controlled was just introduced by the BlockingRequestReply pattern of our first example. Again, adaptation of the pattern divides into renaming of generic identifiers and some minor refinement steps. Note, however, that ReceivingProtocolMachine is not touched at all by the pattern application (see structural diagram of the pattern description, Figure 8), although it is required as part of the context specification. For composition of the pattern instance, it is necessary that the protocol designer gives positioning information, where the pattern elements have to be placed within the embedding context: the timer is set the first time when the message is sent the first time. The timer is reset whenever an expected confirmation has been received. Finally, the states that are prepared to consume a confirmation must also accept and react to a timer signal that triggers the retransmission. It should be mentioned that retransmission may lead to duplication of messages. Therefore, additional SDL patterns have been applied in subsequent design steps that are not discussed here.

\section{COMPARISON OF SDL INHERITANCE AND SDL-PATTERN APPLICATION}

So far, we have listed examples of SDL inheritance and SDL-pattern application. In this section, we will investigate how these concepts are related, and to what degree one concept can be expressed in terms of the other one. First, this will lead to a better understanding of SDL-pattern application, as this new concept can partially be explained based on an existing SDL concept. Second, it indicates a generalization of this SDL concept. The results are summarized in Table 1.

We start with an example where SDL-pattern application can be expressed by SDL specialization. Consider the application of the TimerControlledRepeat pattern, as shown in Figure 8, which results in a modification of the procedure AskReceiver

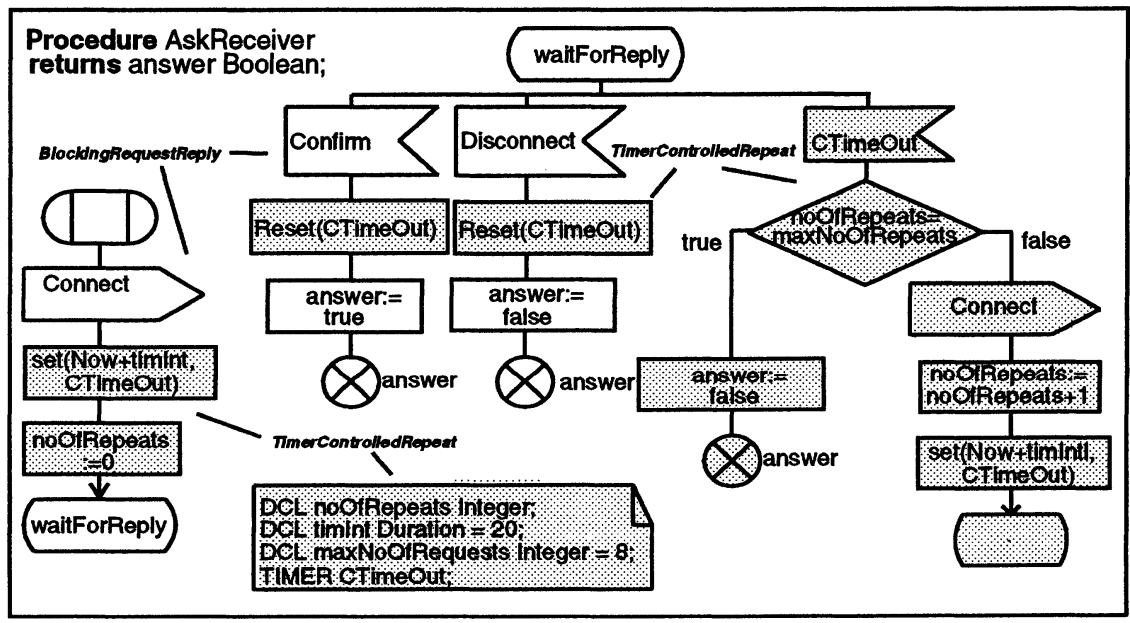

Figure 9. Refined description of procedure AskReceiver after applying the TimerControlledRepeat pattern 
(Figure 9). This modification can be expressed in terms of SDL specialization by redefining the input transitions of the given context (dashed symbols), and by adding another transition that consumes the signal 'timerName' as shown in Figure 9. All that is required is that the redefined input transitions are declared as virtual. The resulting relationship is visualized by the UML diagram in Figure 10, where AskReceiver2 specializes (inherits) AskReceiverl.

Here, the question arises to what degree SDL specialization covers SDL-pattern application in the previous situa-

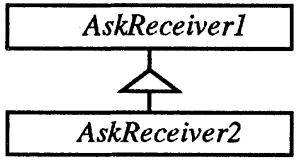

Figure 10. SDL-pattern application in terms of SDL specialization: TimerControlledRepeat tion. If we can express SDL-pattern application as SDL specialization, then this gives rise to a class hierarchy, consisting, on each level, of superclasses and subclasses: in the previous example, AskReceiver 2 is a subclass of AskReceiver1. This class hierarchy documents the incremental nature of the development process; however, as we are interested in the final class description only, and have the information about SDL-pattern application available, intermediate classes may be eliminated.

To what degree can the context of a SDL-pattern application be captured by SDL language constructs? We have noted already that in case of the TimerControlledRepeat pattern, this context can be given by an SDL specification fragment as shown in Figure 8, with virtual input transitions. However, this context is not complete: if we look at the structure item of the pattern, this shows that there has to be another class, which - though it remains unmodified by the pattern application - must have a particular relationship with AskReceiverl.

Based on these observations, we draw a first conclusion, that the syntactical portion of SDL-pattern application can indeed be expressed syntactically by SDL specialization in case of the TimerControlledRepeat pattern, but that there are further aspects that go beyond SDL specialization. This means that we have language support only as far as the syntactic modifications are concerned.

Next, we consider the application of the BlockingRequestReply pattern, shown in Figure 6. Application of this pattern results in the modification of two process types, namely SendingProtocolMachine and ReceivingProtocolMachine. Each of these modifications can again be expressed in terms of SDL specialization, either by redefining an existing transition and adding an attribute (SendingProtocolMachine), or by adding further transitions (ReceivingProtocolMachine). This situation is visualized by the UML diagrams in Figure 11a.

We point out here that the SDL-pattern description requires SendingProtocolMachine and ReceivingProtocolMachine to be modified simultaneously. However, the

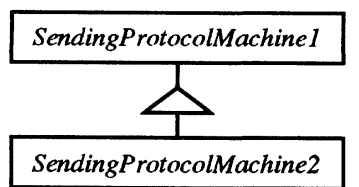

(a)

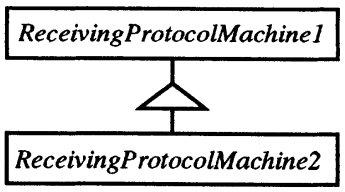

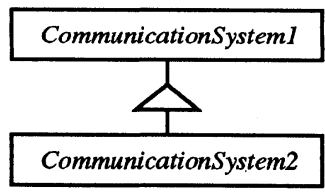

(b)

Figure 11. SDL-pattern application in terms of SDL specialization: BlockingRequestReply 
UML diagrams in Figure 11a are unconnected, meaning that SDL specialization as described here considers each process type independently, which does not reflect the aforementioned constraint. To avoid this problem, one could think of redefining the closest SDL type that contains both process type definitions, i.e., CommunicationSystem. SDL specialization permits to redefine structure of a system type that is declared virtual by replacing the block types suitably. Thus, the application of the BlockingRequestReply pattern can be expressed in terms of SDL specialization by a redefinition on system level, as shown in Figure 11b.

This is not the language support one might expect, as it requires a new specification of the components replacing the block types of CommunicationSysteml, thus obscuring the modifications that have actually been made. These modifications are not on system level, but on process level. Nevertheless, they can be understood as a specialization of the closest SDL type containing the process types. We observe here that this kind of specialization is only indirectly supported by SDL specialization, as explained before. The reason is that SDL specialization is possible only on the upper refinement level of the specialized type, which is not sufficient in the considered case. Therefore, we argue that it would be useful and systematic to generalize the notion of SDL specialization by allowing simultaneous modifications inside the components (blocks, processes, services) of an SDL type. This generalized form of specialization would adequately support the application of most of the SDL patterns that are currently defined in our protocol pattern pool.

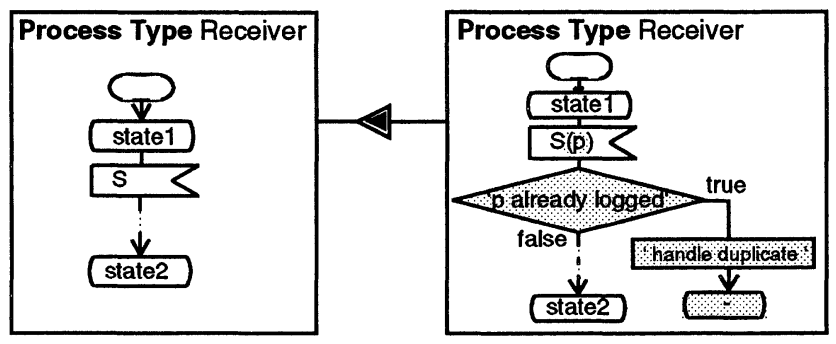

Figure 12.

SDL-pattern application: DuplicateHandle

Another requirement on language support is to modify a process description by adding a new signal parameter to one of the input signals. This is for example necessary for most applications of the SDL pattern DuplicateHandle (see [12] for a description of this pattern). There, an identifier is added to each signal in order to recognize duplicates. Furthermore, an input transition is refined by an additional branch where duplicates are handled. This is illustrated in Figure 12. With the specialization concepts of SDL, adding a new transition branch can be done by redefining the input transition. Adding a signal parameter, however, is much more complicated. In principle, an SDL signal type can be specialized by adding new signal parameters as follows:
signal $S 1$;
$/ *$ definition of signal type S1 without a parameter $* /$
signal $S 2$ inherits $S 1$ adding $(P) ; \quad / *$ signals of type $S 2$ contain a parameter of type $P$ */

However, the SDL specification must then be refined to deal with the signal subtype $S 2$ instead of the signal supertype $S 1$. For instance, all relevant input transitions must 


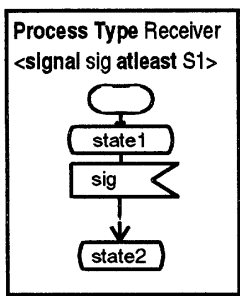

(a)

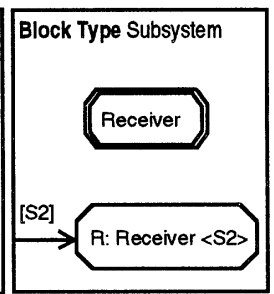

(b)

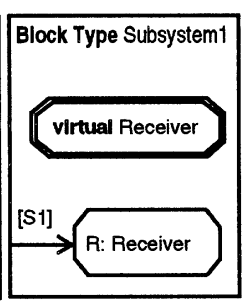

(c)

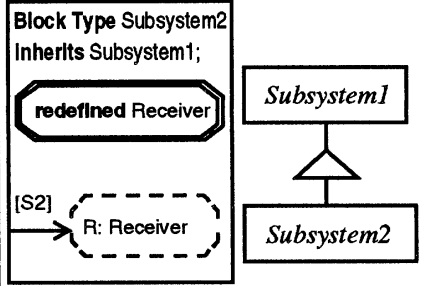

(d)

(e)

Figure 13. SDL-pattern application in terms of SDL specialization: DuplicateHandle

be modified. In order to make an SDL type independent from the context in which it is defined, so-called context parameters are applied. One would expect that parameterizing Receiver with a signal context parameter as shown in Figure 13a would solve the problem. In this case, it would be possible to instantiate Receiver with a subtype of $S 1$, in our case $S 2$ (Figure 13b). However, the additional parameter $p$ of $S 2$ is not accessible in the definition of Receiver (Figure 13a). Therefore, it is not possible to specialize the input transition by adding the additional transition branch. One would have to add the new parameter $p$ also to the signal name in the input symbol. However, this kind of specialization is not supported by SDL. We therefore have to define Receiver as a virtual process type (Figure 13c) and redefine it by specializing the embedding block type (Figures 13d,e).

Another problem appears with architectural SDL patterns such as the DynamicEntitySet pattern [12]. An SDL block type or system type can be specialized by adding new blocks, processes, channels, signals, or gates or by redefining virtual blocks or processes. Other modifications, e.g., removing a process or redefining a channel, are not supported. However, for the DynamicEntitySet pattern it is, for instance, necessary to re-route an existing channel, i.e., to disconnect it first and then connect it to a newly introduced process. It turns out that not all kinds of structural changes are directly supported by SDL specialization. However, the example above can again be modeled by redefining a higher-level block.

Table 1. Comparison of SDL inheritance and SDL-pattern application

\begin{tabular}{|l|l|}
\hline \multicolumn{1}{|c|}{ SDL inheritance } & \multicolumn{1}{|c|}{ SDL-pattern application } \\
\hline \hline $\begin{array}{l}\text { expresses commonalities between types by defin- } \\
\text { ing relations within one specification } \\
\text { (class-based inheritance) }\end{array}$ & $\begin{array}{l}\text { documents incremental development steps by defining } \\
\text { relations between different specifications }\end{array}$ \\
\hline $\begin{array}{l}\text { applies to a single SDL type } \\
\text { specialization only possible on the upper } \\
\text { refinement level of the SDL type }\end{array}$ & $\begin{array}{l}\text { applies to a (possibly empty) set of design elements } \\
\text { (SDL specification units, e.g., types, transitions, signals) }\end{array}$ \\
\hline refinement of transitions cannot be constrained & $\begin{array}{l}\text { simultaneous specialization of different } \\
\text { design elements on all refinement levels possible }\end{array}$ \\
\hline no support for: & refinement of transitions can be constrained \\
\hline - adding new signal parameters \\
re-routing channels and signal routes
\end{tabular}




\section{CONCLUSIONS}

In this paper, we have argued that the application of SDL patterns is a general form of specialization, supporting the reusability of design decisions, and have compared it to the well-known reuse mechanism of inheritance in SDL. As it has turned out, the concepts are related, but are introduced with different objectives. While SDL inheritance aims at expressing commonalities between types within a specification, SDL-pattern application focuses on external reuse of design decisions and documentation of incremental development steps. Nevertheless, we have shown that certain syntactical aspects of pattern application are in some cases expressible by SDL specialization. .However, due to certain language restrictions, we had to use a different relation called design element specialization to cover further cases. Also, pattern application allows one to define constraints under which desirable properties of the resulting SDL specification are achieved. This goes beyond the purely syntactical use of SDL inheritance.

\section{References}

[1] G. Booch, J. Rumbaugh, I. Jacobson. The Unified Modeling Language, Version 1.0. Rational Software Corporation, 1997

[2] F. Buschmann, R. Meunier, H. Rohnert, P. Sommerlad, M. Stal. Pattern-Oriented Software Architecture - A System of Patterns. John Wiley \& Sons, 1996

[3] D. Cisowski, B. Geppert, F. Rößler, M. Schwaiger. Tool Support for SDL Patterns. 1st Workshop of the SDL Forum Society on SDL and MSC, Berlin, Germany, 1998

[4] E. Cusack. Refinement, Conformance and Inheritance. Formal Aspects of Computing, 3: 129-141, 1991

[5] E. Cusack. Inheritance in Object Oriented Z. European Conference on Object Oriented Programming (ECOOP'91), 1991

[6] L. Delgrossi, L. Berger (Ed.). Internet Stream Protocol Version 2 (ST2), Protocol Specification - Version ST2+. RFC 1819, 1995

[7] J. Ellsberger, D. Hogrefe, A. Sarma. SDL - Formal Object-oriented Language for Communicating Systems. Prentice Hall, 1997

[8] E. Gamma, R. Helm, R. Johnson, J. Vlissides. Design Patterns - Elements of Reusable Object-Oriented Software. Addison-Wesley, 1995

[9] B. Geppert, R. Gotzhein, F. Rößler. Configuring Communication Protocols Using SDL Patterns. SDL'97 - Time for Testing - SDL, MSC and Trends, 8th SDL Forum, France, 1997

[10] B. Geppert, A. Kühlmeyer, F. Rößler, M. Schneider. SDL-Pattern based Development of a Communication Subsystem for CAN. IFIP Joint Int. Conf. on Formal Description Techniques \& Protocol Spec., Testing, and Verif., FORTE XI/PSTV XVIII'98, France, 1998

[11] B. Geppert, F. Rößler, Generic Engineering of Communication Protocols - Current Experience and Future Issues. 1st IEEE Int. Conf. on Formal Engineering Methods, ICFEM'97, Hiroshima, Japan, 1997

[12] B. Geppert, F. Rößler. Pattern-based Configuring of a Customized Resource Reservation Protocol with SDL. SFB 501 Report 19/96, Computer Science Department, University of Kaiserslautern, Germany, 1996

[13] R. Gotzhein, G.v. Bochmann. Specialization in Estelle. in: S. T. Vuong, S. T. Chanson (eds.), Protocol Specification, Testing, and Verification XIV, Chapman \& Hall, 1995

[14] ITU-T Recommendation Z.100 (03/93). CCITT Specification and Description Language $(S D L)$. International Telecommunication Union (ITU)

[15] ITU-T Recommendation Z.120 (10/96). Message Sequence Chart (MSC), International Telecommunication Union (ITU)

[16] A. Olsen, O. Færgemand, B. Møller-Pedersen, R. Reed, J. R. W. Smith. Systems Engineering Using SDL-92, Elsevier Science B. V., 1994 